\title{
Characterization of the Shielding Properties of Serum Albumin on a Plant Viral Nanoparticle
}

\author{
Neetu M. Gulati ${ }^{1}$, Andrzej S. Pitek ${ }^{2}$, Nicole F. Steinmetz ${ }^{2,3}$, and Phoebe L. Stewart ${ }^{1}$ \\ 1. Department of Pharmacology and Cleveland Center for Membrane and Structural Biology, Case \\ Western Reserve University, Cleveland, Ohio USA. \\ 2. Department of Biomedical Engineering, Case Western Reserve University, Cleveland, Ohio USA. \\ 3. Departments of Radiology, Macromolecular Science and Engineering, Materials Science and \\ Engineering, and Case Comprehensive Cancer Center, Case Western Reserve University, Cleveland, \\ Ohio USA.
}

Nanoparticle development is an important avenue of research for drug delivery in the field of cancer, in order to deliver a high payload of therapeutic molecules to a tumor site while reducing off-target effects elsewhere in the body. However, a critical barrier for the success of nanoparticle platforms is the rapid elimination of nanoparticles from circulation. To shield nanoparticles from recognition and clearance by the immune system, drug delivery platforms are often coated in polyethylene glycol (PEG), which induces a 'stealth effect'. PEGylation reduces serum protein adsorption on the surface of the nanoparticle and therefore reduces immune detection; however PEGylation also has disadvantages. The effects of PEGylation are dependent on the physical characteristics of the PEG polymer used, including chain length and architecture [1], which may be due to the polymer's flexible and hydrophilic nature. Also, a recent study has shown that as much as $25 \%$ of the population has PEG-specific antibodies, which would allow for enhanced clearance of PEGylated nanoparticles [2]. This is a drastic increase from the less than $1 \%$ of the population measured in a study performed in 1984 [3]. Furthermore, a nanoparticle's behavior in vivo can be altered by the proteins adsorbed to its surface during circulation, a phenomenon known as formation of a protein corona [4,5]. We have recently developed a nanoparticle platform with a manufactured protein corona that allows for shielding from the immune system. Our platform consists of a plant viral nanoparticle, tobacco mosaic virus (TMV), conjugated to serum albumin (SA) through a short PEG linker. SA is the most abundant plasma protein and therefore acts as a camouflage. Our preliminary results have shown that SA shielding reduces antibody recognition of the nanoparticle better than TMV coated with a short PEG linker alone (data not shown). We predict the SA molecules are evenly distributed on the surface of our SA-shielded TMV nanoparticles. Furthermore, we expect that the short PEG linker attaching SA to the TMV surface does not allow much flexibility of the SA molecules. Therefore, we hypothesize that antibodies are unable to penetrate the SA layer in order to interact with the TMV nanoparticle surface. In this study, the physical properties of SA on the surface of TMV are characterized to better understand the mechanism of immune shielding.

TMV nanoparticles are produced through infection of Nicotiana benthamiana plants as described previously [5]. Purified nanoparticles are conjugated to SA with a short PEG linker through NHS and maleamide chemistries. Conjugation to TMV is confirmed via SDS-PAGE analysis. Purified particles are characterized for antibody recognition through immunogold labeling and negative stain electron microscopy. Further characterization of SA on the surface of TMV is done through cryo-electron microscopy (cryoEM) and tomography (cryoET) to visualize coverage of TMV and the distribution of the shielding protein on the surface of TMV. CryoEM grids are imaged on a JEOL 2200FS 200kV (FEG, energy filter) transmission cryo-electron microscope with a DE20 direct electron detector. 
Our results indicate that SA effectively reduces antibody recognition of TMV nanoparticles as compared to bare TMV or TMV-PEG. This is presumably due to well-distributed SA proteins on the surface of TMV as shown by cryoEM images (Figure 1). CryoET studies will provide more detailed 3D information on SA coverage of the TMV surface. Further studies will investigate alternative SA attachments to TMV, including longer PEG linkers and varying coverage levels.

References:

[1] KL Lee et al, Acta Biomaterialia 19 (2015), p. 166.

[2] RP Garay et al, Expert Opin Drug Deliv 9 (2012), p. 1319.

[3] AW Richter and E Akerblom, Int Arch Allergy Appl Immunol, 74 (1984), p. 36.

[4] D Walczyk et al, J Am Chem Soc, 132 (2010), p. 5761

[5] AS Pitek et al, Small (2016), epub. ahead of print.

[6] MA Bruckman and NF Steinmetz, Methods Mol Biol 1108 (2014), p. 173.
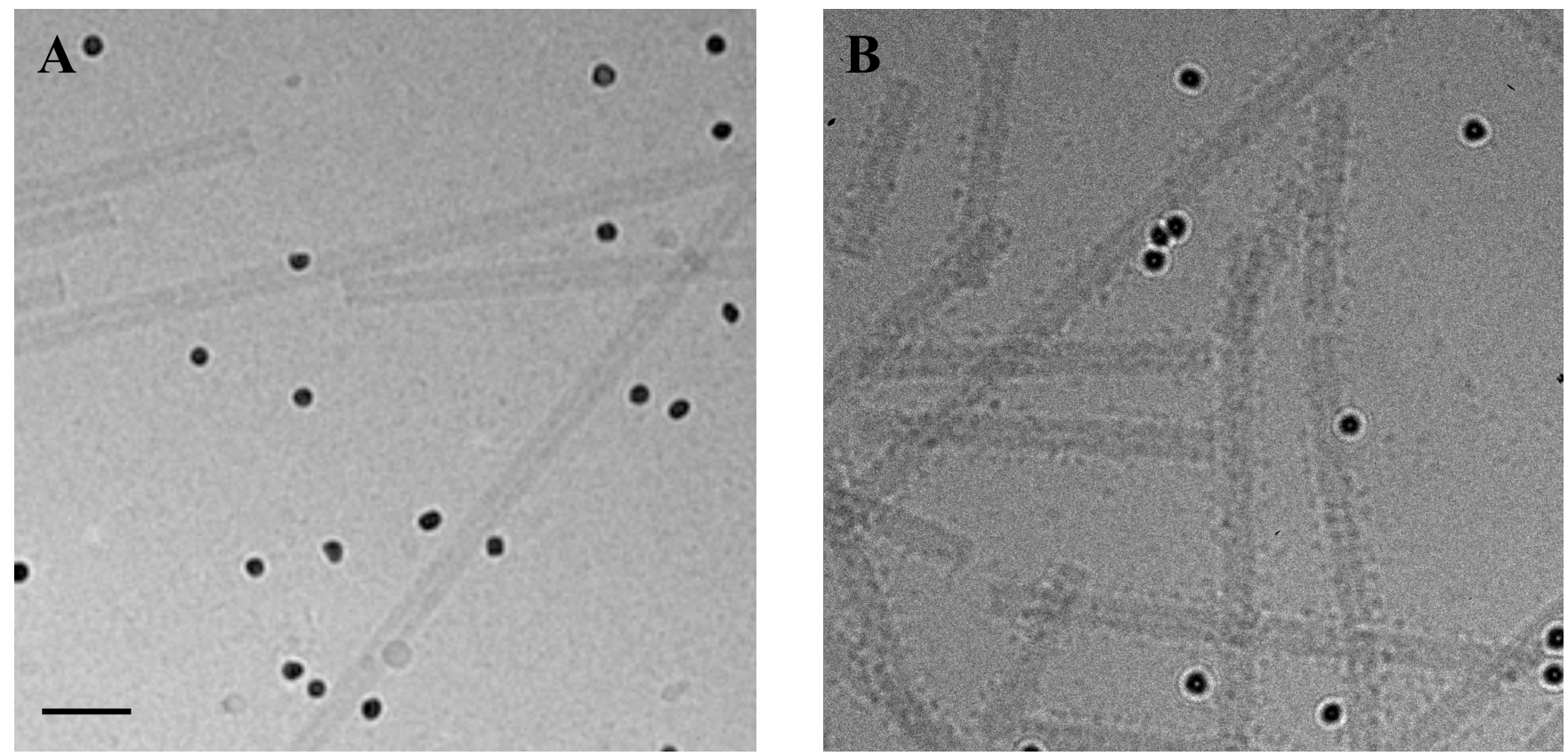

Figure 1. Sections of cryo-electron micrographs of A) bare TMV nanoparticles and B) TMV nanoparticles conjugated to SA via a short (8-mer) PEG linker. As compared to the bare TMV nanoparticles, TMV-SA particles show protrusions all along the length of the rod shaped TMV, indicative of well-distributed SA conjugated to the surface of the nanoparticles. Scale bar is $25 \mathrm{~nm}$. Black circles are 10nm gold fiducials. 